\title{
GLOBAL SOLUTIONS AND DECAY OF A NON LINEAR COUPLED SYSTEM WITH THERMO-ELASTIC
}

\author{
Ricardo Fuentes Apolaya ${ }^{1}$, Raúl Izaguirre Maguiña ${ }^{2}$.
}

(Recibido: 24/07/2015 - Aceptado: 20/08/2015)

$$
\begin{aligned}
& \text { Abstract: In this present work, the authors prove the existence of global solutions } \\
& \text { and the decay of nonlinear wave equation with thermo-elastic coupling give by the } \\
& \text { system of equation: } \\
& \qquad u^{\prime \prime}(x, t)-\mu(t) \Delta u(x, t)+\sum_{i=1}^{n} \frac{\partial \theta}{\partial x_{i}}(x, t)+F(u(x, t))=0 \text { in } Q=\Omega \times(0, \infty) \\
& \qquad \theta^{\prime}(x, t)-\Delta \theta(x, t)+\sum_{i=1}^{n} \frac{\partial u^{\prime}}{\partial x_{i}}(x, t)=0 \text { in } Q=\Omega \times(0, \infty),
\end{aligned}
$$

where $\mathrm{u}$ is displacement, $\theta$ is absolute temperature, $\Delta$ denotes the Laplace operator, $\mu$ is a positive real function of $t, F: \mathbb{R} \rightarrow \mathbb{R}$ is continuous function such that $s \cdot F(s) \geq 0, \Omega$ is a smooth bounded open set in $\mathbb{R}^{n}$ with boundary $\Gamma$.

Keywords: Weak solutions; Strauss approximation; asymptotic behavior.

\section{Introduction}

The nonlinear wave equation with thermos-elastic coupling is given by the system of equation

$$
\begin{gathered}
u^{\prime \prime}(x, t)-\mu(t) \Delta u(x, t)+\sum_{i=1}^{n} \frac{\partial \theta}{\partial x_{i}}(x, t)+F(u(x, t))=0 \\
\theta^{\prime}(x, t)-\Delta \theta(x, t)+\sum_{i=1}^{n} \frac{\partial u^{\prime}}{\partial x_{i}}(x, t)=0
\end{gathered}
$$

with initial and boundary conditions

$$
\begin{gathered}
u(x, 0)=u^{0}(x), \quad u^{\prime}(x, 0)=u^{1}(x), \quad \theta(x, 0)=\theta^{0}(x) \text { in } \Omega, \\
u(x, t)=0, \quad \theta(x, t)=0 \text { in } \Gamma \times(0, \infty),
\end{gathered}
$$

where $\mathrm{u}$ is displacement, $\theta$ is absolute temperature, $\Delta$ denotes the Laplace operator, $\mu$ is a positive real function of $\mathrm{t}, \mathrm{F}$ is a function such that $s \cdot F(s) \geq 0$, the temporal partial derivative is represented by $\frac{\partial u}{\partial t}=u^{\prime}$, is $\Omega$ is a smooth bounded open set in $\mathbb{R}^{n}$ with $C^{2}$ boundary $\Gamma$, and $Q=\Omega \times(0, \infty)$.

The non linearity $F(v)=|v|^{\rho} v$ usually appears in relativistic quantum mechanic (see Segal [13] or Schiff [12]), and has been considered by various authors for hyperbolic, parabolic and elliptic equations. Lions [6] studied the wave equation with the same non linearity, i.e., $|v|^{\rho} v$, in

\footnotetext{
${ }^{1}$ Universidade Federal Fluminense, IM, RJ, Brasil, e-mail: ricardof16@yahoo.com.br

${ }^{2}$ UNMSM, Facultad de Ciencias Matemáticas, e-mail: raul_izaguirre2222@yahoo.es
} 
a smooth bounded open domain $\Omega$ of $\mathbb{R}^{n}$ and proved existence and uniqueness of solution using both Faedo-Galerkin's and Compactnesss' methods.

In [1] investigated the system (1) - (4) with $F(v)=|v|^{\rho} v$. They established global existence and strong and weak solutions by Faedo-Galerkin's method using a basis of the space $H_{0}^{1}(\Omega) \cap H^{2}(\Omega)$ and the exponential stability of total energy associated to the weak solution using KomornikZuazua's method [4].

Based in the theory developed in the paper [1] and Strauss approximations of F [15], we will prove that the system (1) - (4) has a unique global strong solution, a unique global weak solution, and the total energy associated to these solutions is asymptotically stable.

The outline of this chapter is as follows. In Section 2, the basic theory is laid out and global existence of strong and weak solutions are issued for the Lipschitzian case and general case, whilst exponential decay in Section 3.

\section{Existence of Solution}

To obtain the existence and uniqueness of global solution of the mixed problem (1) - (4) we suppose the additional hypotheses about $\mu$ and the function $\mathrm{F}$ :

$$
\mu \in W_{l o c}^{1,1}(0, \infty), \quad \mu(t) \geq \mu_{0}, \forall t \geq 0 \text { and } \mu^{\prime}(t) \leq 0 \text { a. e. in }(0, \infty)
$$

$\mathrm{F}$ is continuous and $s \cdot F(s) \geq 0$ for all $s \in \mathbb{R}$

Let us represent by $G$ the function

$$
G(s)=\int_{0}^{s} F(r) d r
$$

Now we can present the existence results of the initial and boundary value problem (1) - (4).

Theorem 2.1 (Case: F Lipchitzian ) Let $F: \mathbb{R} \rightarrow \mathbb{R}$, be such that $s \cdot F(s) \geq 0$, $F$ is Lipschitzian and derivable except a finite number of points and $\mu$ be the function defined by above hypothesis (5). Given

$$
u_{0}, \theta_{0} \in H_{0}^{1}(\Omega) \cap H^{2}(\Omega) \quad, u_{1} \in H_{0}^{1}(\Omega)
$$

then the system (1) - (4) has a unique strong solution $\{u, \theta\}$ such that

$$
\begin{gathered}
u, \theta \in L^{\infty}\left(0, \infty ; H_{0}^{1}(\Omega)\right) \\
u^{\prime} \in L^{\infty}\left(0, \infty ; L^{2}(\Omega)\right) \cap L_{l o c}^{\infty}\left(0, \infty ; H_{0}^{1}(\Omega)\right), \quad \theta^{\prime} \in L_{l o c}^{2}\left(0, \infty ; H_{0}^{1}(\Omega)\right) \\
u^{\prime \prime} \in L_{l o c}^{\infty}\left(0, \infty ; L^{2}(\Omega)\right)
\end{gathered}
$$

Proof: Existence. To show global existence of solution we will use both the Faedo-Galerkin's and Compactness' methods. We consider $\left(w_{j}\right)_{j \in \mathbb{N}}$ an orthonormal basis of $H_{0}^{1}(\Omega) \cap H^{2}(\Omega)$, and denote by $V_{m}=\left[w_{1}, w_{2}, \ldots, w_{m}\right]$ the subspace of $H_{0}^{1}(\Omega) \cap H^{2}(\Omega)$ spanned by the $\mathrm{m}$ first vectors of $\left(w_{j}\right)_{j \in \mathbb{N}}$.

In these conditions, the approximated system associated to system (1) - (2) is given by

$$
\begin{gathered}
u_{m}(t)=\sum_{i=1}^{m} g_{j m}(t) w_{j}, \quad \theta_{m}(t)=\sum_{i=1}^{m} h_{j m}(t) w_{j} \\
\left(u_{m}^{\prime \prime}(t), v\right)+\mu(t)\left(\left(u_{m}(t), v\right)\right)+\sum_{i=1}^{n}\left(\frac{\partial \theta_{m}}{\partial x_{i}}(t), v\right)+\left(F\left(u_{m}(t)\right), v\right)=0
\end{gathered}
$$




$$
\left(\theta_{m}^{\prime}(t), w\right)+\left(\left(\theta_{m}(t), w\right)\right)+\sum_{i=1}^{n}\left(\frac{\partial u_{m}^{\prime}}{\partial x_{i}}(t), w\right)=0
$$

where $\mathrm{v}$ and w belong to $V_{m}$.

Let $u_{m}(0)=u_{0 m}, u_{m}^{\prime}(0)=u_{1 m}$ and $\theta_{m}(0)=\theta_{0 m}$ be. Hence $u_{0 m}, u_{1 m}$ and $\theta_{0 m}$ belong to $V_{m}$ and satisfy

$$
\begin{gathered}
u_{0 m} \rightarrow u_{0} \text { strongly in } H_{0}^{1}(\Omega) \cap H^{2}(\Omega) \\
\theta_{0 m} \rightarrow \theta_{0} \text { strongly in } H_{0}^{1}(\Omega) \cap H^{2}(\Omega) \\
u_{1 m} \rightarrow u_{1} \text { strongly in } H_{0}^{1}(\Omega)
\end{gathered}
$$

Under these conditions, the system (6) - (7) has a local solution $\left\{u_{m}(t), \theta_{m}(t)\right\}$ over the interval $\left[0, t_{m}\right)$. This interval will be extended to any interval $[0, \infty)$ thanks to the first estimate below.

Estimate I. Substituting v by $2 u_{m}^{\prime}(t)$ and w by $2 \theta_{m}(t)$ in (6) and (7) respectively, using Green's formula in the term $\sum_{i=1}^{n}\left(\frac{\partial u_{m}^{\prime}}{\partial x_{i}}(t), \theta\right)$ and integrating over $[0, t), 0 \leq t \leq t_{m}$, we get

$$
\begin{gathered}
2\left(u_{m}^{\prime \prime}(t), u_{m}^{\prime}(t)\right)+2 \mu(t)\left(\left(u_{m}(t), u_{m}^{\prime}(t)\right)\right)+2 \sum_{i=1}^{n}\left(\frac{\partial \theta_{m}}{\partial x_{i}}(t), u_{m}^{\prime}(t)\right) \\
+2\left(F\left(u_{m}(t)\right), u_{m}^{\prime}(t)\right)=0 \\
2\left(\theta_{m}^{\prime}(t), \theta_{m}(t)\right)+2\left(\left(\theta_{m}(t), \theta_{m}(t)\right)\right)+2 \sum_{i=1}^{n}\left(\frac{\partial u_{m}^{\prime}}{\partial x_{i}}(t), \theta_{m}(t)\right)=0
\end{gathered}
$$

We obtain

$$
\begin{gathered}
\frac{d}{d t}\left[\left|u_{m}^{\prime}(t)\right|^{2}+\left|\theta_{m}(t)\right|^{2}+\mu(t)\left\|u_{m}(t)\right\|^{2}\right]+ \\
+2 \int_{\Omega} F\left(u_{m}(t)\right) \cdot u_{m}^{\prime}(t) d x+2\left\|\theta_{m}(t)\right\|^{2}=\mu^{\prime}(t)\left\|u_{m}(t)\right\|^{2}
\end{gathered}
$$

We denote

$$
E_{1 m}(t)=\left|u_{m}^{\prime}(t)\right|^{2}+\left|\theta_{m}(t)\right|^{2}+\mu(t)\left\|u_{m}(t)\right\|^{2}+2 \int_{\Omega} G\left(u_{m}(t)\right) d x
$$

Integrating de 0 a $t<t_{m}$, we obtain:

$$
E_{1 m}(t)+2 \int_{0}^{t}\left\|\theta_{m}(s)\right\|^{2} d s=E_{1 m}(0)+2 \int_{0}^{t} \mu^{\prime}(s)\left\|u_{m}(s)\right\|^{2} d s
$$

We know that

$$
u_{0 m} \rightarrow u_{0} \text { in } H_{0}^{1}(\Omega) \cap H^{2}(\Omega) \subset L^{2}(\Omega)
$$

Then we have

$$
\int_{\Omega} G\left(u_{0 m}\right) d x \rightarrow \int_{\Omega} G\left(u_{0}\right) d x
$$

By convergence there is a positive constant $K_{1}$, independent of $\mathrm{m}$ such that

$$
E_{1 m}(t)+2 \int_{0}^{t}\left\|\theta_{m}(s)\right\|^{2} d s \leq K_{1} \text { for all } t \geq 0
$$

Hence, we can extent the approximate solutions $\left\{u_{m}(t), \theta_{m}(t)\right\}$ on the whole interval $[0, \infty)$ independent of $\mathrm{m}$ and $\mathrm{t}$.

Estimative II. Make sense take the first derivative of the approximated equation because the existence theorem implied that $u_{m}^{\prime \prime}$ is absolutely continuous on $[0, T]$. Derive both sides with respect to $t$

$$
\left(u_{m}^{\prime \prime \prime}(t), v\right)+\mu(t)\left(\left(u_{m}^{\prime}(t), v\right)\right)+\sum_{i=1}^{n}\left(\frac{\partial \theta_{m}^{\prime}}{\partial x_{i}}(t), v\right)+
$$




$$
\begin{gathered}
+\left(F^{\prime}\left(u_{m}(t)\right) u_{m}^{\prime}(t), v\right)=-\mu^{\prime}(t)\left(\left(u_{m}(t), v\right)\right) \\
\left(\theta_{m}^{\prime \prime}(t), w\right)+\left(\left(\theta_{m}^{\prime}(t), w\right)\right)+\sum_{i=1}^{n}\left(\frac{\partial u_{m}^{\prime \prime}}{\partial x_{i}}(t), w\right)=0
\end{gathered}
$$

We denote

$$
E_{2 m}(t)=\left|u_{m}^{\prime \prime}(t)\right|^{2}+\mu(t)\left\|u_{m}^{\prime}(t)\right\|^{2}+\left|\theta_{m}^{\prime}(t)\right|^{2}
$$

Take $v=2 u_{m}^{\prime \prime}(t)$ and $w=2 \theta^{\prime}(t)$, we get

$$
\begin{aligned}
& \frac{d}{d t} E_{2 m}(t)+2\left\|\theta_{m}^{\prime}(t)\right\|^{2} \leq 2 \beta\left|u_{m}^{\prime}(t)\right| \cdot\left|u_{m}^{\prime \prime}(t)\right|+2 \frac{\mu^{\prime}(t)}{\mu(t)}\left|u_{m}^{\prime \prime}(t)\right|^{2} \\
& 2 \frac{\mu^{\prime}(t)}{\mu(t)} \sum_{i=1}^{n} \int_{\Omega} \frac{\partial \theta_{m}}{\partial x_{i}}(t) u_{m}^{\prime \prime}(t) d x+2 \frac{\mu^{\prime}(t)}{\mu(t)} \int_{\Omega} F\left(u_{m}(t)\right) \cdot u_{m}^{\prime \prime}(t) d x
\end{aligned}
$$

By hypotheses of the $\mu$, and usual inequalities yields applying the Gronwall's inequality we obtain and

$$
E_{2 m}(t)+2 \int_{0}^{t}\left\|\theta_{m}^{\prime}(s)\right\|^{2} d s \leq K_{2} \text { for all } t \geq 0
$$

The regularity for $u$ and $\theta$ is guaranteed by results on elliptic regularity (see, for instance, Medeiros and Milla Miranda [9] or Nirenberg [11].

Theorem 2.2 (Weak Solution) Given $u_{0}, \theta_{0} \in H_{0}^{1}(\Omega), G\left(u_{0}\right) \in L^{1}(\Omega)$ and $u_{1} \in L^{2}(\Omega)$ then system (1.1)-(1.4) has a unique weak solution $\{u, \theta\}$ such that

$$
\begin{gathered}
u, \theta \in L^{\infty}\left(0, \infty ; H_{0}^{1}(\Omega)\right) \\
u^{\prime} \in L^{\infty}\left(0, \infty ; L^{2}(\Omega)\right), \quad \theta^{\prime} \in L^{2}\left(0, \infty ; L^{2}(\Omega)\right)
\end{gathered}
$$

and equations (1.1) and (1.2) are given in the sense of $L^{\infty}\left(0, \infty ; L^{2}(\Omega)\right)$.

Proof: We use arguments of density.

Theorem 2.3 (General Case) Let $F: \mathbb{R} \rightarrow \mathbb{R}$, be continuous such that $s \cdot F(s) \geq 0$.

Consider

$$
G(s)=\int_{0}^{s} F(\sigma) d \sigma
$$

Given

$$
u_{0}, \theta_{0} \in H_{0}^{1}(\Omega), \quad G\left(u_{0}\right) \in L^{1}(\Omega) \text { and } u_{1} \in L^{2}(\Omega)
$$

then there exists $\{u, \theta\}: Q \rightarrow \mathbb{R}$ such that:

$$
\begin{gathered}
u, \theta \in L^{\infty}\left(0, \infty ; H_{0}^{1}(\Omega)\right) \\
u^{\prime} \in L^{\infty}\left(0, \infty ; L^{2}(\Omega)\right), \quad \theta^{\prime} \in L^{2}\left(0, \infty ; L^{2}(\Omega)\right)
\end{gathered}
$$

and $\{u, \theta\}$ satisfies the equations

$$
\begin{gathered}
u^{\prime \prime}(x, t)-\mu(t) \Delta u(x, t)+\sum_{i=1}^{n} \frac{\partial \theta}{\partial x_{i}}(x, t)+F(u(x, t))=0, \quad \text { in } L_{l o c}^{\infty}\left(0, \infty, L^{2}(\Omega)\right) \\
\theta^{\prime}(x, t)-\Delta \theta(x, t)+\sum_{i=1}^{n} \frac{\partial u^{\prime}}{\partial x_{i}}(x, t)=0, \quad \text { in } L_{l o c}^{\infty}\left(0, \infty, L^{2}(\Omega)\right)
\end{gathered}
$$

and initial conditions

$$
u(0)=u_{0}, \quad u^{\prime}(0)=u_{1}, \quad \theta(0)=\theta_{0}
$$


Proof: We first approximate $u_{0}$ by a sequence of functions $\left(u_{0 j}\right)_{j \in \mathbb{N}}$ of $H_{0}^{1}(\Omega) \cap L^{\infty}(\Omega)$. In fact, let us consider

$$
\beta_{j}(s)= \begin{cases}s, & \text { if }|s| \leq j \\ j, & \text { if } s>j \\ -j, & \text { if } s<-j\end{cases}
$$

Use the notation $\beta_{j}\left(u_{0}\right)=u_{0 j}$. Then $u_{0 j} \in H_{0}^{!}(\Omega)$ (see Brezis-Cazanave [2]) and $u_{0 j} \rightarrow u_{0} \in$ $H_{0}^{1}(\Omega)$.

Let $F$ and $G$ be as above and represent by $F_{k}$ the Strauss approximation of $F$, that is, $F_{k}, k \in \mathbb{N}$, is a continuous function defined by:

$$
\begin{cases}F_{k}(s)=-(k)\left[G\left(s-\frac{1}{k}\right)-G(s)\right] & \text { if } \quad-k \leq s \leq-\frac{1}{k} \\ F_{k}(s)=k\left[G\left(s+\frac{1}{k}\right)-G(s)\right] & \text { if } \frac{1}{k} \leq s \leq k \\ F_{k}(s) \text { is linear by parts } & \text { if }-\frac{1}{k}<s \leq \frac{1}{k} \text { with } F_{k}(0)=0 \\ F_{k}(s) \text { appropriate constants } & \text { for }|s|>k .\end{cases}
$$

It follows by Strauss [15], that $F_{k}$ is Lipschitz for each $k$,

$s F_{k}(s) \geq 0$ and $\left(F_{k}\right)$ converges to $F$ uniformly on the compacts subsets of $\mathbb{R}$.

Represent by

$$
G_{k}(s)=\int_{0}^{s} F_{k}(r) d r, \quad G_{k}(0)=F_{k}(0)=0, \quad s . G_{k}(s) \geq 0
$$

for all $k \in \mathbb{N}$.

Approximations of $u_{0 j}, \theta_{0}$ and $u_{1}$ by elements of $\mathfrak{D}(\Omega)$.

Let $\varphi_{\nu j} \in \mathfrak{D}(\Omega), \chi_{\nu} \in \mathfrak{D}(\Omega)$ and $\psi_{\nu} \in \mathfrak{D}(\Omega)$ such that

$$
\begin{gathered}
\varphi_{\nu j} \rightarrow u_{0 j} \text { in } H_{0}^{1}(\Omega) \\
\chi_{\nu} \rightarrow \theta_{0} \text { in } H_{0}^{1}(\Omega) \\
\psi_{\nu} \rightarrow u_{1} \text { in } L^{2}(\Omega)
\end{gathered}
$$

Then, by Theorem above, Lipschitz case, there exists a unique $\left\{u_{\nu j k}, \theta_{\nu k}\right\}$ in the conditions:

$$
\begin{gathered}
u_{\nu j k} \in L^{\infty}\left(0, \infty ; H_{0}^{1}(\Omega)\right) \cap L_{l o c}^{\infty}\left(0, \infty, H_{0}^{1}(\Omega) \cap H^{2}(\Omega)\right) \\
\theta_{\nu k} \in L^{\infty}\left(0, \infty ; L^{2}(\Omega)\right) \cap L^{2}\left(0, \infty, H_{0}^{1}(\Omega)\right) \\
u_{\nu j k}^{\prime} \in L^{\infty}\left(0, \infty ; L^{2}(\Omega)\right) \cap L_{l o c}^{\infty}\left(0, \infty, H_{0}^{1}(\Omega)\right) \\
\theta_{\nu k}^{\prime} \in L_{l o c}^{\infty}\left(0, \infty ; L^{2}(\Omega)\right) \cap L_{l o c}^{2}\left(0, \infty, H_{0}^{1}(\Omega)\right) \\
u_{\nu j k}^{\prime \prime} \in L_{l o c}^{\infty}\left(0, \infty ; L^{2}(\Omega)\right) \\
\left(u_{\nu j k}^{\prime \prime}(t), v\right)+\mu(t) a\left(u_{\nu j k}(t), v\right)+\sum_{i=1}^{n}\left(\frac{\partial \theta_{\nu k}}{\partial x_{i}}(x, t), v\right)+\left(F\left(u_{\nu j k}(t)\right), v\right)=0 \\
\left(\theta_{\nu k}^{\prime}(t), w\right)+a\left(\theta_{\nu k}(t), w\right)+\sum_{i=1}^{n}\left(\frac{\partial u_{\nu j k}^{\prime}}{\partial x_{i}}(t), w\right)=0 \\
u_{\nu j k}(0)=\varphi_{\nu j}, \quad u_{\nu j k}^{\prime}(0)=\psi_{\nu}, \quad \theta_{\nu k}(0)=\chi_{\nu} .
\end{gathered}
$$

By similar arguments used to obtain estimates, we find

$$
\left|u_{\nu j k}^{\prime}(t)\right|^{2}+\left|\theta_{\nu k}(t)\right|^{2}+\mu(t)\left\|u_{\nu j k}(t)\right\|^{2}+2 \int_{\Omega} G_{k}\left(u_{\nu j k}(t)\right) d x+2 \int_{0}^{t}\left\|\theta_{\nu k}(s)\right\|^{2} d s \leq
$$




$$
\leq\left|\psi_{\nu}\right|^{2}+\left|\chi_{\nu}\right|^{2}+\mu(0)\left\|\varphi_{\nu j}\right\|^{2}+2 \int_{\Omega} G_{k}\left(\varphi_{\nu j}\right) d x
$$

By the above convergences of $\left(\psi_{\nu}\right),\left(\varphi_{\nu j}\right)$ and $\left(\chi_{\nu}\right)$ we obtain that the second member of the preceding inequality is bounded except $\int_{\Omega} G_{k}\left(\varphi_{\nu j}\right) d x$.

Therefore the behavior of Estimative I depends on the behavior of the term $\int_{\Omega} G_{k}\left(\varphi_{\nu j}\right) d x$ as $\nu \rightarrow \infty$. We must estimate independent of $\mathrm{j}$ and $\mathrm{k}$ too.

We divide the proof in three parts.

First Part We prove that

$$
\int_{\Omega} G_{k}\left(\varphi_{\nu j}\right) d x \rightarrow \int_{\Omega} G_{k}\left(u_{0 j}(x)\right) d x, \nu \rightarrow \infty
$$

By convergence there exists a subsequence of $\left(\varphi_{\nu j}\right)$, still denoted by $\left(\varphi_{\nu j}\right)$, and a function $v_{j} \in L^{2}(\Omega)$ such that

$$
\begin{gathered}
\varphi_{\nu j}(x) \rightarrow u_{0 j}(x) \text { a.e. in } \Omega, \nu \rightarrow \infty \\
\left|\varphi_{\nu j}(x)\right| \leq v_{j}(x) \text { a.e. in } \Omega,
\end{gathered}
$$

By continuity of $G_{k}(s)$, we obtain

$$
G_{k}\left(\varphi_{\nu j}(x)\right) \rightarrow G_{k}\left(u_{0 j}(x)\right), \nu \rightarrow \infty
$$

Let $c_{k}$ be the Lipschitz constant of $F_{k}(s)$. We have

$$
0 \leq G_{k}\left(\varphi_{\nu j}(x)\right) \leq c_{k}\left|\varphi_{\nu j}(x)\right| \leq c_{k} v_{j}(x) \text { a.e. in } \Omega
$$

The above two convergences and Lebesgue Theorem of Dominated Convergence imply first part.

Second Part We show that

$$
\int_{\Omega} G\left(u_{0 j}(x)\right) d x \rightarrow \int_{\Omega} G\left(u_{0}(x)\right) d x, \quad j \rightarrow \infty
$$

In fact, by the continuity of $G(s)$ and convergence,

$$
G\left(u_{0 j}(x)\right) \rightarrow G\left(u_{0}(x)\right) \text { a.e. in } \Omega, j \rightarrow \infty
$$

By construction of $u_{0 j}$ we find

$$
G\left(u_{0 j}(x)\right) \leq G\left(u_{0}(x)\right) \text { a.e. in } \Omega, \forall j
$$

Noting that $G\left(u_{0}\right) \in L^{1}(\Omega)$, the last two expressions and Lebesgue Theorem of Dominated Convergence, give convergence of second part.

Third Part We prove that

$$
\int_{\Omega} G_{k}\left(u_{0 j}(x)\right) d x \rightarrow \int_{\Omega} G\left(u_{0 j}(x)\right) d x, \quad k \rightarrow \infty
$$

In fact, for $\mathrm{j}$ fixed we obtain

$$
\left|u_{0 j}(x)\right| \leq j, \text { a.e. in } \Omega
$$

We note that

$$
G_{k}^{\prime}(s)=F_{k}(s) \rightarrow G^{\prime}(s)=F(s) \text { uniformly in }[-j, j]
$$

Also

$$
G_{k}(0)=0 \rightarrow G(0)=0
$$


Then

$$
G_{k}(s) \rightarrow G(s) \text { uniformly in }[-j, j]
$$

In particular

$$
G_{k}\left(u_{0 j}(x)\right) \rightarrow G\left(u_{0 j}(x)\right) \text { uniformly in } \Omega, k \rightarrow \infty
$$

This implies convergence third part.

Uniqueness: We use arguments of density, Strauss approximation and energy inequality.

\section{Asymptotic Behavior of Solutions}

In order to obtain the decay of solutions we introduce an internal damping in the problem, more precisely, we consider the following system:

$$
\begin{array}{ll}
u^{\prime \prime}(x, t)-\mu(t) \Delta u(x, t)+\sum_{i=1}^{n} \frac{\partial \theta}{\partial x_{i}}(x, t)+F(u(x, t))+\gamma u^{\prime}(x, t)=0, & \text { in } Q=\Omega \times(0, \infty) \\
\theta^{\prime}(x, t)-\Delta \theta(x, t)+\sum_{i=1}^{n} \frac{\partial u^{\prime}}{\partial x_{i}}(x, t)=0, & \text { in } Q=\Omega \times(0, \infty)
\end{array}
$$

where $\gamma$ is a positive constant.

We make the supplementary hypothesis

$$
s F(s) \geq C_{0} G(s), \quad \forall s \in \mathbb{R}\left(C_{0} \text { positive constant }\right)
$$

With the same hypothesis on $\mathrm{F}(\mathrm{s})$ and initial data $u_{0}, u_{1}, \theta_{0}$ and by similar arguments used to obtain Theorem (3.1) and Theorem (4.1) .

We get, respectively, strong solutions and weak solutions of the mixed problem for system (14). Consider the energy

$$
E(t)=\left|u^{\prime}(t)\right|^{2}+|\theta(t)|^{2}+\mu(t)\|u(t)\|^{2}+2 \int_{\Omega} G(u(t)) d x, t \geq 0
$$

associated to system (14).

By applying a Lyapunov functional, we obtain the follwing result:

Theorem 3.1 (Asymptotic Behavior) Assume hypotheses of Theorem (4.1) and hypotheses (15). Let $\{u, \theta\}$ be the solution given by Theorem (4.1) for system (14). Then

$$
E(t) \leq 3 E(0) e^{-\eta t}, \quad t \geq 0
$$

where $\eta=\min \left\{\epsilon_{1}, \epsilon_{3}\right\}>0$ 


\section{REFERENCIAS BIBLIOGRÁFICAS}

[1] Apolaya R. F., Clark H. R., Feitosa A. J. On a nonlinear coupled system with internal damping. Electronic Journal of Differential Equations, Volume 200064 (2000), pp.1-17.

[2] Brezis H., Cazenave T., Non Linear Evolution Equations. Lecture Notes at. Instituto de Matemática, UFRJ, Rio de Janeiro, RJ, Brasil. (1994).

[3] Clark H. R., Jutuca San Gil L. P..Milla Miranda M. On a mixed problem for a linear coupled system with variable coefficients. Electronic Journal of Differential Equations, Volume 104 (1998), pp.1-20.

[4] Komornik, V., Zuazua E. A direct method for boundary stabilization of the wave equation. J. Math. Pure et Appl., Volume 69, (1990), pp. 33-54.

[5] Ladyzhenskaia O. A.,Visik M. I. Boundary value problems for partial differential equations and certain classes of operator equations. A. M. S. Translations Series 210 (1958). pp. 223-281..

[6] Lions, J.L. Quelques méthodes de résolution des problèmes aux limites non linéares, DunodGauthier Villars, Paris, First edition, (1969).

[7] Lions, J.L., Magenes, E. Non-homogeneous boundary value problems and applications. Vols. 1, Springer-Verlag, New York, (1972).

[8] Medeiros L. A.,Milla Miranda M. On a boundary value problem for wave equations: Existence, uniquenesse-asymptotic behavior. Revista de Matemáticas Aplicadas, Universidad de Chile, 17, (1996) pp. 47-73.

[9] Medeiros L. A.,Milla Miranda M. Espaço de Sobolev - Iniciação aos problemas elípticos não homogêneos . Instituto de Matemática - UFRJ, Rio de Janeiro, First Edition, (1977).

[10] Milla Miranda M.Medeiros L. A., Hidden regularity for Semilinear Hyperbolic Partial Differential Equations. An. Fac. des Sciences de Tolouse, Volume IX 01 (1988) pp. 103-120.

[11] Nirenberg L., Remarks on strongly elliptic partial differential equations. Comm. Pure Applied Math., 08, (1977) pp. 648-674.

[12] Schiff L. I., Non linear meson theory of nuclear forces. I. Physic. Rev., 84, (1951) pp. 1-9.

[13] Segal I. E., The global Cauchy problem for a relativistic scalar field with power interaction. Bull. Soc. Math., France 91, (1963) pp. 129-135.

[14] Sobolev S. I., Applications de analyse functionnelle aux équations de la physique mathématique. Lêningrad, (1950)

[15] Strauss W. A., On weak solutions of semilinear hyperbolic equations. An. Acad. Bras. Ciências Volume 42, 04 (1950), pp. 645-651. 